Research Article

\title{
Multiagent Consensus Control Strategy considering Whole-Process Thermodynamic Characteristics of Air Conditioning Process
}

\author{
Jun Li, ${ }^{1}$ Bo Geng, ${ }^{1}$ Zhixian Lin, ${ }^{1}$ Min Chen, ${ }^{1}$ Liangyou Shao, ${ }^{1}$ Yanmin Zhou, ${ }^{2}$ \\ and Yuqing Bao $\mathbb{1}^{2}$ \\ ${ }^{1}$ Shenzhen Power Supply Bureau Co. Ltd., Shenzhen 518000, China \\ ${ }^{2}$ School of Electrical Engineering, Southeast University, Nanjing 210096, China \\ Correspondence should be addressed to Yuqing Bao; 503000747@qq.com
}

Received 22 February 2021; Accepted 20 July 2021; Published 29 July 2021

Academic Editor: James Lam

Copyright (C) 2021 Jun Li et al. This is an open access article distributed under the Creative Commons Attribution License, which permits unrestricted use, distribution, and reproduction in any medium, provided the original work is properly cited.

\begin{abstract}
Due to the distributed and decentralized characteristics of air conditioning load, the distributed control strategy has advantages for the air conditioning load to participate in the demand response. However, existing approaches focus on the dynamic control performance with very few considerations on the cost. To fill this gap, this paper proposes a multiagent consensus control method considering the whole-process response cost of air conditioning. Based on the thermodynamic characteristics of air conditioning load in the load reduction process and recovery process, the cost function curve of air conditioning load is established. Then, the multiagent consensus control strategy is adopted to send the power adjustment information to each air conditioner to realize the optimal control of the air conditioning load. The simulation results verify that the proposed method can take into account the whole-process response cost of air conditioning loads and result in smaller control cost than existing methods.
\end{abstract}

\section{Introduction}

With the increasing penetration of renewable energy, it is increasingly difficult for the power system to keep the active power balance by using generation side resources. More and more attention has been paid to the utilization of demand side resources to accommodate renewable energy.

As an important demand side resource, air conditioning load occupies a relatively high proportion in the power load and has the capacity of cold and hot storage. Focusing on the control strategy of air conditioning load, a lot of research works have been reported. Bao et al. [1] proposed an air conditioning load control strategy based on the state-queue model, through the broadcast control signal to achieve load tracking control of large number of air conditioning loads. In [2], a centralized control strategy based on supply-demand interaction is proposed, in which the household energy management device is used to send load shedding signals to the air conditioning load, and the large-scale air conditioning loads are controlled. In [3], a direct load control strategy of inverter air conditioners is proposed, which can control the air conditioning group by changing the set-point of air conditioning temperature. In [4], a direct load control method based on fuzzy logic is proposed, and a fuzzy controller with triangular membership function is designed to control the air conditioning load. In [5], a direct load control strategy based on Fourier transform is proposed to track the load demand curve of central air conditioning.

Due to the distributed characteristics of the air conditioning loads, the traditional centralized control method has problems in sending command to large amount of nodes. Conversely, the distributed control framework has advantages in sending control signals and improving the control system reliability. In recent years, many distributed control methods have been proposed, for example, the nonnegative edge consensus control method [6], the positivity-preserving consensus control method [7], and the event-triggered guaranteed cost consensus control method [8]. The 
distributed consensus method is applied to energy storage systems [9], the microgrids [10], the distributed generators [11], and so on.

Focusing on the distributed control strategy of air conditioning loads, Wang et al. [12] proposed a consensus control strategy of inverter air conditioner based on the distributed control framework. The adjustable temperature interval was selected as consensus variable and the same degree of control was realized for all the users. In [13], a distributed control strategy of air conditioning load for the large-scale photovoltaic-penetrated system is proposed. By designing a hierarchical distributed control strategy, the dual regulation of power and voltage is realized. Although the distributed consensus control strategy for air-conditioning load is designed in $[12,13]$, the control cost is not considered for the thermodynamic characteristics of air-conditioning load.

Table 1 gives a comparison of the abovementioned methods. It can be seen that none of them consider the whole-process cost of air conditioners. Since the indoor temperature is affected by the load control of air conditioning loads, it is necessary to investigate the whole-process cost according to the thermodynamic characteristics of air conditioning load. By this way, system operators can selectively regulate the air conditioning load with less negative impact, so as to minimize the impact of load control on user's comfort during the control process.

Aiming at the distributed control problem of air conditioning load, this paper proposes a multiagent consensus control method that considers the thermodynamic characteristics of the whole process of air-conditioning load which is proposed to minimize the cost function while achieving the control target, so as to reduce the impact on user's comfort. The contributions of this paper can be summarized as follows:

(1) Establishing a whole-process cost function considering the thermodynamics of air conditioners

(2) Applying the multiagent consensus control method to air conditioners in order to minimize the overall cost of the control

The arrangement of this paper is as follows. Section 2 gives the control strategy of air conditioning loads. In Section 3 , the case study is analyzed. Section 4 is the conclusion of this paper.

\section{Control Strategy of the Air-Conditioning Loads}

2.1. Overall Framework of the Proposed Method. The framework of the proposed method is shown in Figure 1. Firstly, the equivalent thermal parameter (ETP) model of the air conditioners is established. Based on the ETP model, a whole-process control cost function of the air conditioner is defined according to the temperature deviation during the 2 processes, including the load reduction process and the recovery process. To simplify the cost function, the scatter plot of the control cost is sampled and the polynomial function is used to fit the scatter plot. Then, the multiagent consensus cooperative control algorithm is applied to the control problem, in order to get the optimal allocation of the adjustment power.

2.2. Thermodynamic Model of Air Conditioning Load. The thermodynamic model of a single air conditioner can be represented by an ETP model, as shown in Figure 2. It can be expressed as follows:

$$
\dot{T}_{a}=-\frac{1}{\mathrm{RC}} T_{a}+\frac{1}{\mathrm{RC}} T_{o} \pm \frac{\eta}{C} P,
$$

where $T_{\mathrm{a}}$ is the air temperature in the room, $T_{\mathrm{o}}$ is the outdoor temperature, $R$ is the thermal resistance of the room, $C$ is the heat capacity of the room, $P$ is the electrical power of air conditioning load, and $\eta$ is the thermal efficiency of air conditioning load. The plus and minus sign in front of $\eta$ depends on whether the air conditioner is in the cooling or heating mode. The negative sign is taken when the air conditioner is in the cooling mode, and the positive sign is taken when the air conditioner is in the heating mode.

For fixed-frequency air conditioners, the power cannot be continuously adjusted. The power $P$ of the air conditioner is a discrete variable, taking 0 or $P_{\max }$. The power control is realized by duty cycle control, and its dynamic process is relatively complex. For inverter air conditioners, the power can be continuously adjusted within $\left[0, P_{\max }\right]$. In this paper, a distributed control strategy is designed for the inverter air conditioner whose power can be adjusted continuously.

2.3. Establishment of Whole-Process Cost Function of Air Conditioning Load. Based on the equivalent thermal parameter model of air conditioning load, a cost function model considering the whole-process thermodynamic characteristics of air conditioning load is established through sampling and fitting.

On the basis of the air conditioning parameters $R, C$, and $\eta$, the parameters indoor temperature $T_{\mathrm{a}}$, outdoor temperature $T_{\mathrm{o}}$, and initial air conditioning power $P$ are initialized according to the actual scene. Under initial conditions, the indoor temperature $T_{\mathrm{a}}$ stabilizes around the initial temperature set point $T_{\text {set } 0}$. When the load-curtailment event occurs, the air conditioning power $P$ and the room temperature $T_{\mathrm{a}}$ will go through 2 processes, as shown in Figure 3:

(i) Load reduction process: in order to respond to the control signal, the air conditioning load reduces the electrical load and the power has a step change of $P_{\mathrm{dec}}$. As a result, the indoor temperature continues to rise.

(ii) Recovery process: after the load reduction, the electric power of air conditioning load is increased to $P=P_{\max }$ in order to restore the room temperature as soon as possible, until the temperature reduces to the initial temperature set point $T_{\text {set0 }}$.

In the whole process, the indoor temperature rises first and then decreases, which has an effect on the comfort of 
TABLE 1: Summary of the abovementioned methods.

\begin{tabular}{lccc}
\hline Methods & Consensus algorithm? & For air conditioners? & Considering the whole-process cost of air conditioners? \\
\hline$[1-5]$ & $\times$ & $\sqrt{ }$ & $\times$ \\
{$[6-11]$} & $\sqrt{ }$ & $\times$ & $\times$ \\
{$[12,13]$} & $\sqrt{ }$ & $\sqrt{ }$ & $\times$ \\
\hline
\end{tabular}

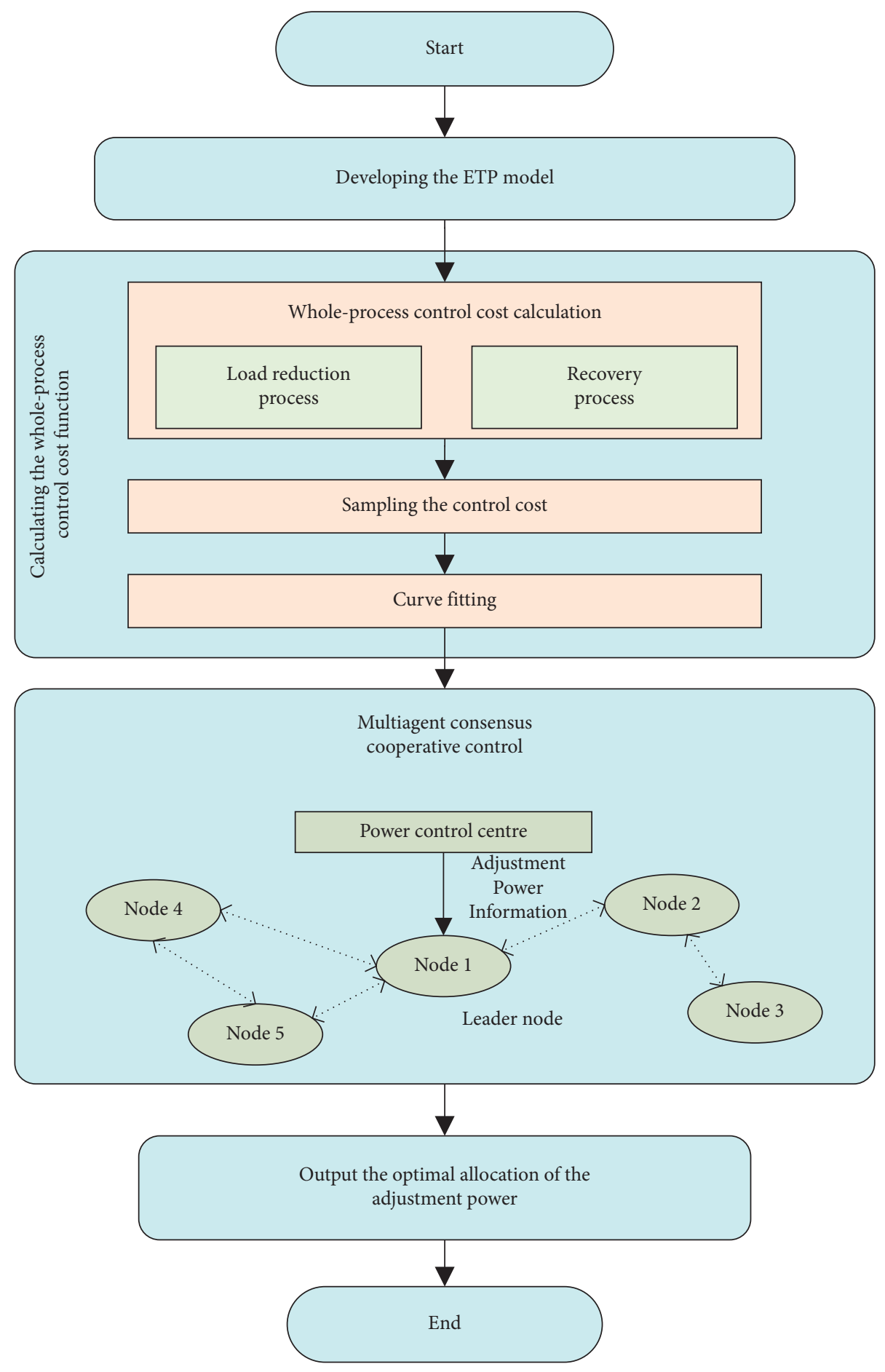

FIGURE 1: The overall flowchart of the proposed method. 


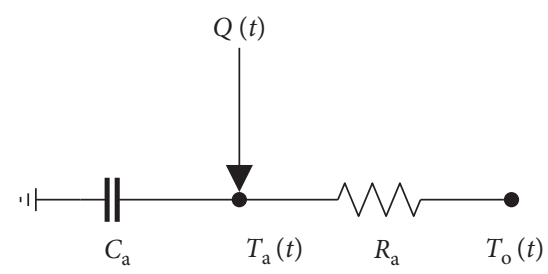

FIGURE 2: equivalent thermal parameter model of single air conditioner.

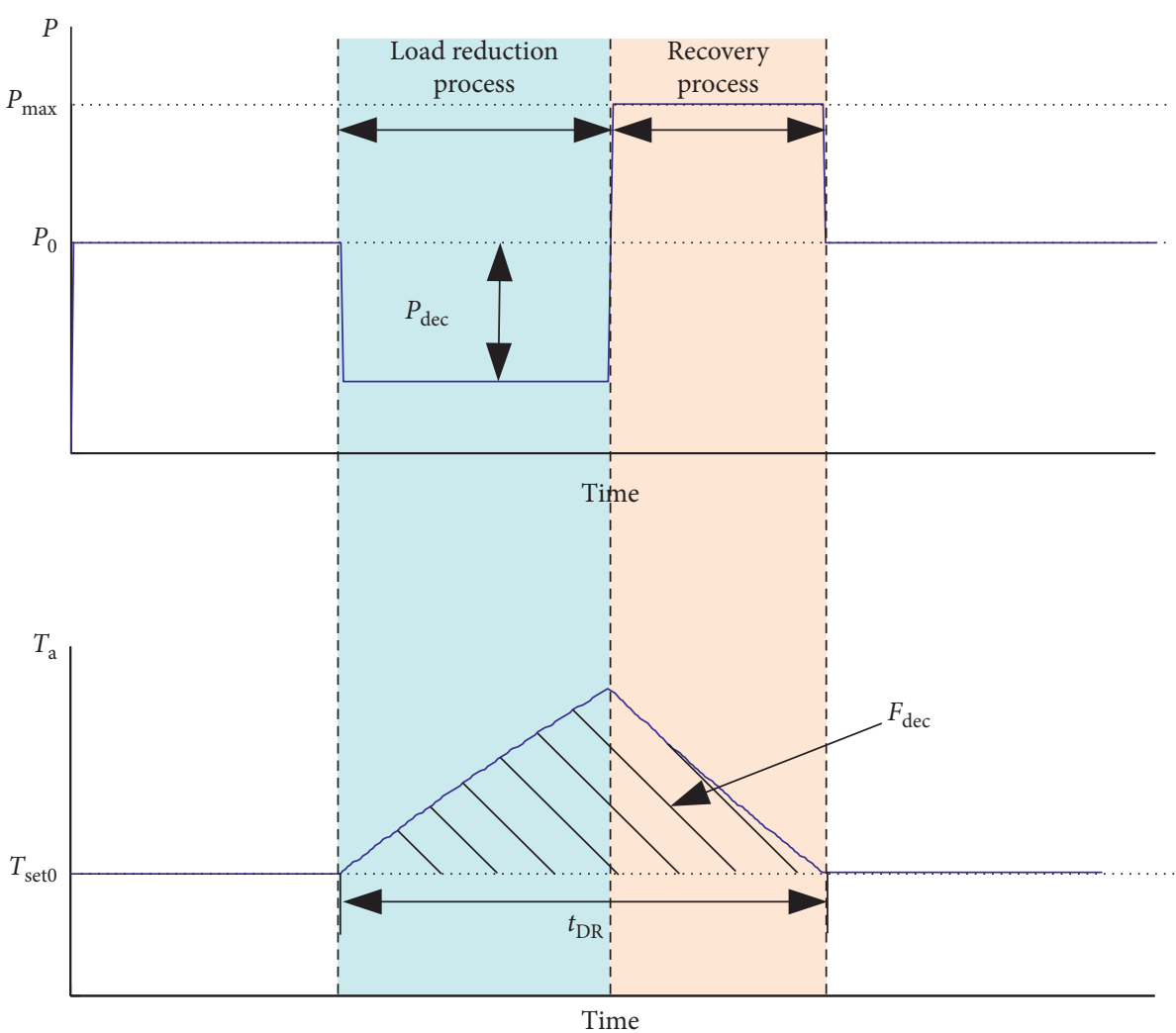

FIgURE 3: Example of air conditioning load control and reduction process and recovery process.

users. The degree of influence is related to the amplitude and duration of temperature deviation. Therefore, the control cost $F_{\mathrm{dec}}$ of the air conditioning load can be defined as the square integral of the indoor temperature deviation $T_{a}-T_{\text {set0 }}$, which can be expressed as

$$
F_{\mathrm{dec}}=\int_{t_{0}}^{t_{0}+t_{D R}}\left(T_{a}-T_{\text {set } 0}\right)^{2} \mathrm{~d} t,
$$

where $t_{0}$ and $t_{\mathrm{DR}}$ are the start time of load reduction event and the duration of temperature deviation, respectively.

In order to establish the cost function of the air-conditioning load, the $P_{\mathrm{dec}}-F_{\mathrm{dec}}$ scatter plot is formed by calculating $F_{\mathrm{dec}}$ under different $P_{\mathrm{dec}}$ conditions. A polynomial function is used to fit the $P_{\mathrm{dec}}-F_{\mathrm{dec}}$ scatter plot, and the following fitting curve is obtained:

$$
F_{\mathrm{dec}}\left(P_{\mathrm{dec}}\right)=a_{m} P_{\mathrm{dec}}^{m}+a_{m-1} P_{\mathrm{dec}}^{m-1}+\cdots+a_{1} P_{\mathrm{dec}}+a_{0},
$$

where $F_{\text {dec }}$ function is used as the basis of multiagent consensus control.
2.4. Multiagent Consensus Cooperative Control. Based on the cost function curve of each air conditioner, the multiagent consensus control strategy is adopted to realize the cooperative control of a large number of air conditioners, so as to achieve the goal of optimal total cost.

Suppose the cost function of the ith air conditioner can be expressed as

$$
F_{\text {deci }}\left(P_{\text {deci }}\right)=a_{m} P_{\text {deci }}^{m}+a_{m-1} P_{\text {deci }}^{m-1}+\cdots+a_{1} P_{\text {deci }}+a_{0},
$$

where $F_{\mathrm{dec} i}\left(P_{\mathrm{dec} i}\right)$ represents the cost of the $i$ th air conditioner, $P_{\text {deci }}$ represents the power reduction of the $i$ th air conditioner, and $a_{m}-a_{0}$ is the corresponding coefficient.

The goal of multiagent control is to minimize the total cost $F_{\text {dec_all }}$ which is expressed as follows:

$$
\operatorname{Min} F_{\text {dec_all }}=\sum_{i} F_{\text {deci }}
$$


In the consensus algorithm, incremental cost is taken as the consensus variable, and the partial derivative of air conditioning cost to its load power is defined as the incremental cost of the load aggregator. For any load aggregator $i$, its incremental cost is

$$
\mathrm{IC}_{i}=\frac{\partial F_{\text {deci }}}{\partial P_{\text {deci }}}=\lambda_{i}, \quad i=1,2,3, \ldots, n .
$$

For the nonleader node, the information update of air conditioning $i$ is realized by the following formula:

$$
\lambda_{i}(k+1)=\sum_{j=1}^{n} \delta_{i j} \lambda_{j}(k), \quad i=1,2,3, \ldots, n,
$$

where $\lambda_{i}$ represents the state information (incremental cost) of air conditioner $i, \lambda_{j}$ represents the state information (incremental cost) of the air conditioner adjacent to air conditioner $i, n$ is the number of air conditioners, and $\delta_{i j}$ represents the element in the sparse iteration matrix and is defined by the following formula:

$$
\delta i j=\frac{\left|l_{i j}\right|}{\sum_{j=1}^{n} l_{i j}}, \quad i=1,2,3, \ldots, n,
$$

where $l_{i j}$ is the corresponding element of the Laplacian matrix.

For the leader node, the air conditioning load $i$ needs to determine whether the reduced load power reaches a given value, and the power adjustment signal needs to be sent to other nodes through the leader node. Therefore, the information update of the leader node air-conditioning load $i$ is realized by the following formula:

$$
\lambda_{i}(k+1)=\sum_{j=1}^{n} \delta_{i j} \lambda_{j}(k)+\varepsilon \Delta P,
$$

where $\varepsilon$ is a positive coefficient called a convergence coefficient, which controls the convergence speed of the leader node, and $\Delta P$ is the total amount of power deviation, which can be expressed as the form of the deviation between the load reduction command and the actual load reduction power of the air conditioning loads:

$$
\Delta P=P_{\text {dec_req }}-\sum_{i=1}^{n} P_{\text {deci }},
$$

where $P_{\text {dec_req }}$ is the power reduction command of all the air conditioners.

Based on equations (4) and (6), the response power $P_{\text {deci }}$ of the air conditioner $i$ can be obtained. The expression of $P_{\mathrm{dec} i}$ depends on the order of the cost function. When the second-order cost function is used, $P_{\text {deci }}$ is calculated by the following formula:

$$
P_{\text {deci }}=\frac{\lambda_{i}-a_{1}}{2 a_{2}} .
$$

When the third-order cost function is used, $P_{\mathrm{dec} i}$ can be calculated as

$$
P_{\mathrm{dec} i}=\frac{-2 a_{2}+\sqrt{4 a_{2}^{2}-12 a_{3}\left(a_{1}-\lambda_{i}\right)}}{6 a_{3}} .
$$

It is difficult to get the expression of $P_{\mathrm{dec} i}$ directly when the cost function is a quartic function or a multiple function of more than four degrees.

Under the iteration of the above algorithm, the incremental cost $\lambda_{i}$ and the power deviation $\Delta P$ tend to converge gradually. Finally, the incremental cost $\lambda_{i}$ of all air conditioners reaches to consensus, and $\Delta P$ tends to zero.

\section{Testing Results}

The example considers a distributed air-conditioning control system of $6 \times 4$ in a building, as shown in Figure 4, with six floors and four occupants (air conditioning) on each floor and assumes that each air-conditioning controller can communicate with the air-conditioning controller of adjacent nodes (upstairs or next door) via a narrow-band Internet, which can form a $6 \times 4$ grid-shaped communication topology. Suppose that the node at the edge of the 1st floor is the leader node and can receive the regulation commands directly. The mean values of the considered air conditioners' parameters are listed in Table 2.

In order to implement distributed control, the cost function of single air conditioning load is established first. In order to determine the order of the polynomial fitting function, the $P_{\mathrm{dec}}-F_{\mathrm{dec}}$ scatter plot and its fitting curves are shown in Figures 5(a) and 5(b). The integral square error (ISE) between the fitting curve and the $P_{\mathrm{dec}}-F_{\mathrm{dec}}$ scatter are shown in Figure 5(c). It can be seen from Figure 5 that, with the increase of the order of polynomial fitting functions, the fitting error shows a downward trend. However, the increase of the order will lead to the complexity of the fitting function, which will increase the cost of parameter identification and distributed calculation.

Using the third-order polynomial function to fit the cost of each air conditioner in the air-conditioning control system, the fitting function results of randomly selected 5 air conditioners are shown in Figure 6. It can be seen that the third-order polynomial function is able to describe the control cost of air conditioning load well.

Figure 7 shows the $P_{\mathrm{dec}}-F_{\mathrm{dec}}$ scatter plot and its fitting curve when the outdoor temperature $T_{\mathrm{o}}$ is different. From Figure 7, it can be seen that, with the change of outdoor temperature $T_{\mathrm{o}}$, the $P_{\mathrm{dec}}-F_{\mathrm{dec}}$ scatter plot and its fitting curve are also different. The higher the outdoor temperature is, the larger the response capacity of air conditioning load can be provided, but the response cost is also increased. In addition, when $T_{\mathrm{o}}$ is different, the third-order polynomial function can fit $P_{\mathrm{dec}}-F_{\mathrm{dec}}$ well, indicating that the fitting function has good robustness.

Then, the effectiveness of the consensus control strategy in this paper is verified. In order to carry out comparative analysis, three distributed control methods are considered:

Method 1 (distributed consensus control strategy): load reduction power is selected as the consensus variable, and the average distribution of the adjustment power can be realized. 


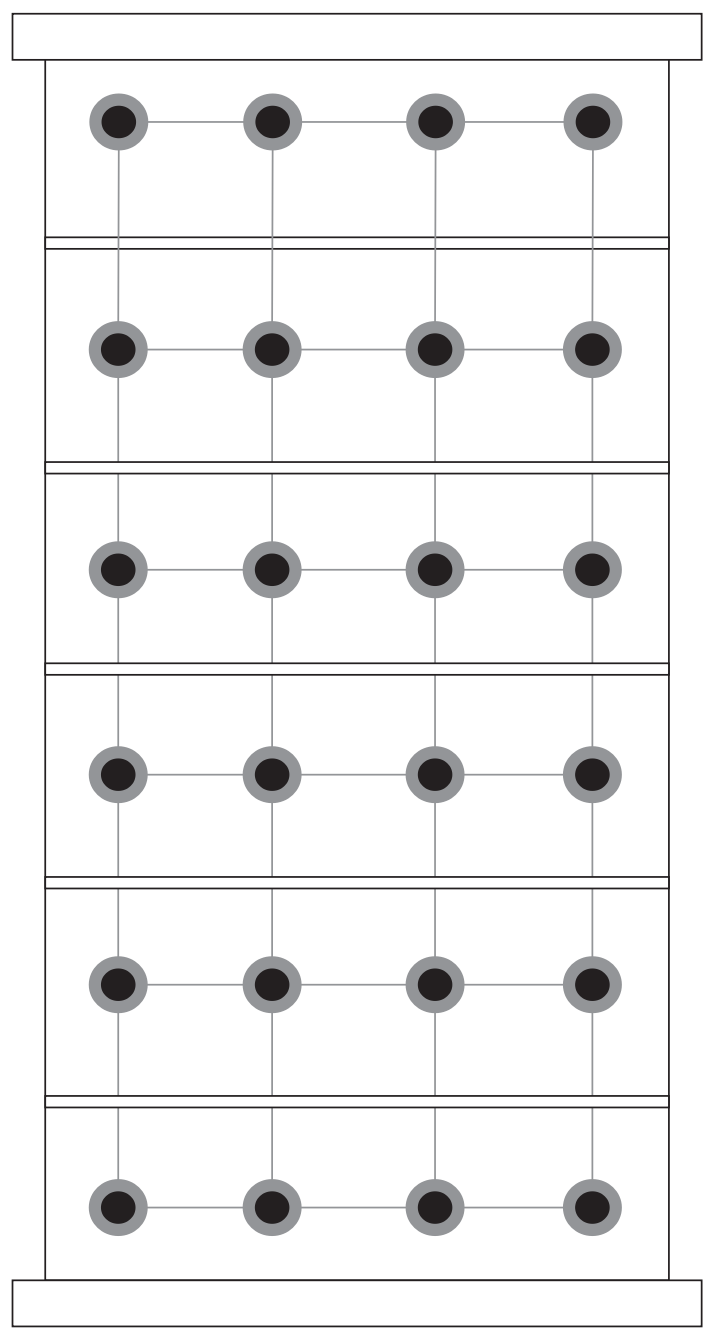

FIGURE 4: Communication topology of the air-conditioning controller.

TABLE 2: Selection of air-conditioning parameters.

\begin{tabular}{lc}
\hline Parameters & Mean value \\
\hline$R$ & $2{ }^{\circ} \mathrm{C} / \mathrm{kW}$ \\
$C$ & $2 \mathrm{kWh} /{ }^{\circ} \mathrm{C}$ \\
$\eta$ & 2.5 \\
$T_{\mathrm{o}}$ & $32^{\circ} \mathrm{C}$ \\
$T_{\text {set } 0}$ & $26^{\circ} \mathrm{C}$ \\
\hline
\end{tabular}

Method 2 (distributed consensus control strategy): the required proportion of load reduction is selected as the consensus variable, and the load reduction is in proportion to the original power consumption of the air conditioning load. This strategy follows the idea of the existing methods $[13,14]$.

Method 3 (the proposed method) (distributed consensus control strategy): the incremental cost of air conditioning load is selected as the consensus variable, and the optimal allocation of the adjustment power can be realized.

Consider the load-curtailment event with $28 \mathrm{~kW}$ power reduction command on a duration of one hour. The simulation results of methods $1 \sim 3$ are shown in Figures 8-10, respectively.

Table 3 lists the total control cost $F_{\text {dec_all }}$ of the three methods and integral absolute error (IAE) of power reduction error $\Delta P$, which is expressed by the following formula:

$$
\mathrm{IAE}=\int_{t_{0}}^{t_{0}+t_{D R}}\left(P_{\text {dec_req }}-\sum_{i=1}^{n} P_{\text {deci }}\right) \mathrm{d} t .
$$

IAE reflects the performance of control. The smaller the IAE is, the better control performance is.

It can be seen from the simulation results that the three control methods have achieved good results in tracking the load reduction signal and achieved the goal of reducing $28 \mathrm{~kW}$ power between $1 \mathrm{~h}$ and $2 \mathrm{~h}$. The IAEs of the three methods are 0.1120, 0.1115, and 0.1047, respectively. However, from the aspect of control cost, method 1 and method 2 does not optimize the cost in the process of control (the cost is 29.2038 and 29.5383), while method 3 (the method in this paper) can achieve the optimal cost through the consensus of the incremental cost (the cost is 28.5434) 

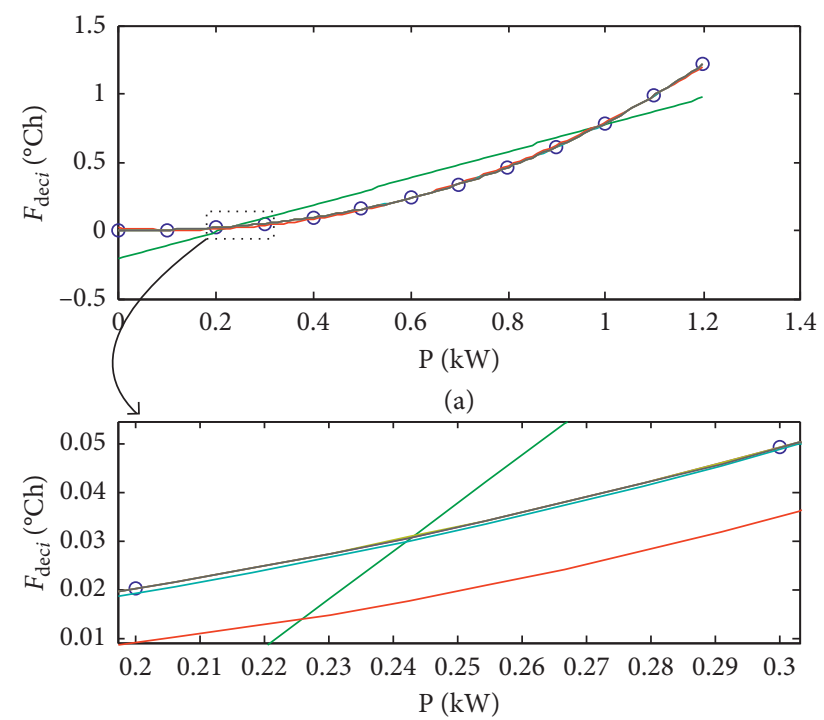

(b)

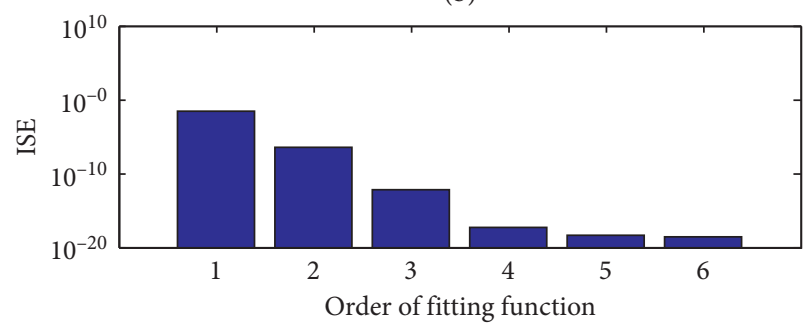

(c)

- Data samples

- Fitting curve ( $1^{\text {st }}$ order function)

- Fitting curve ( $2^{\text {nd }}$ order function)

— Fitting curve ( $3^{\text {rd }}$ order function)

- Fitting curve ( $4^{\text {th }}$ function)

Fitting curve ( $5^{\text {th }}$ function)

- Fitting curve ( $6^{\text {th }}$ function)

FIGURE 5: Fitting results of the cost $F_{\text {dec }}$ with different orders. (a) Adjustment cost fitting curve with different orders of fitting function. (b) Adjustment cost fitting curve with different orders of fitting function (zoomed in). (c) ISE with different orders of fitting function.

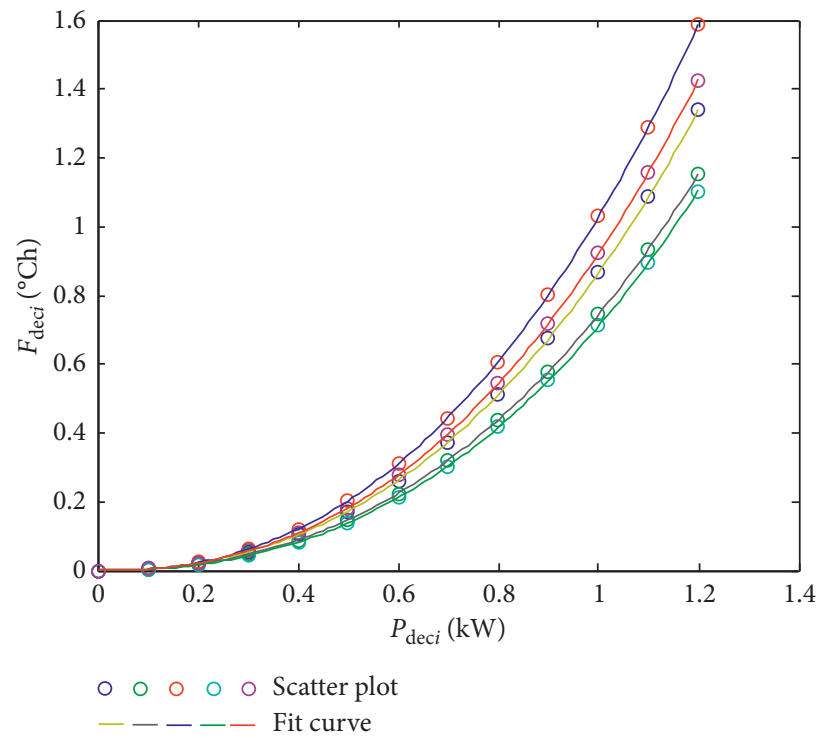

Figure 6: Scatter plot and fit curve of $P_{\mathrm{dec}}-F_{\mathrm{dec}}$ of randomly selected 5 ACs. 


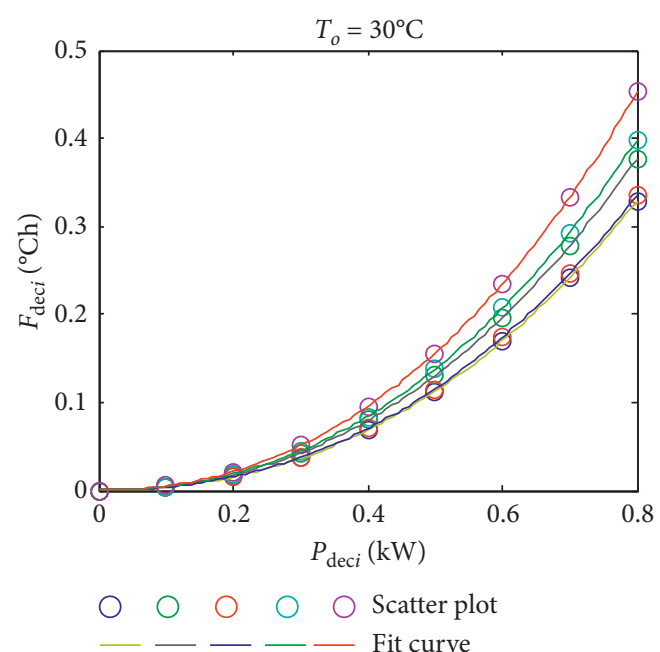

(a)

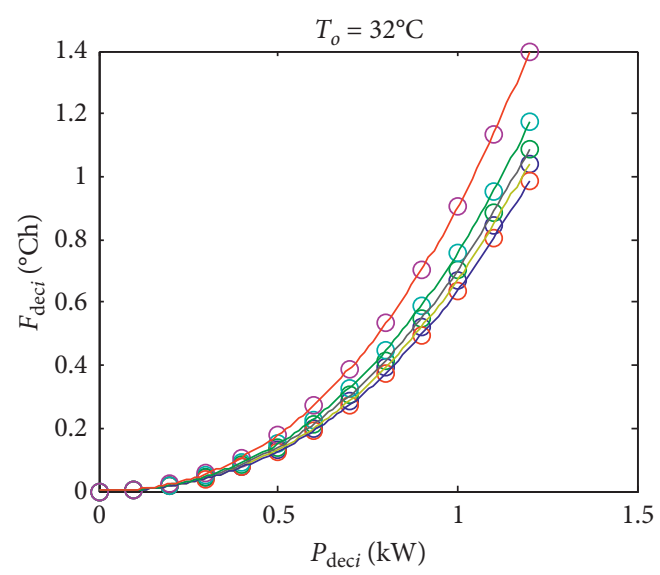

$\bigcirc \bigcirc \bigcirc \bigcirc \bigcirc$ Scatter plot

(c)
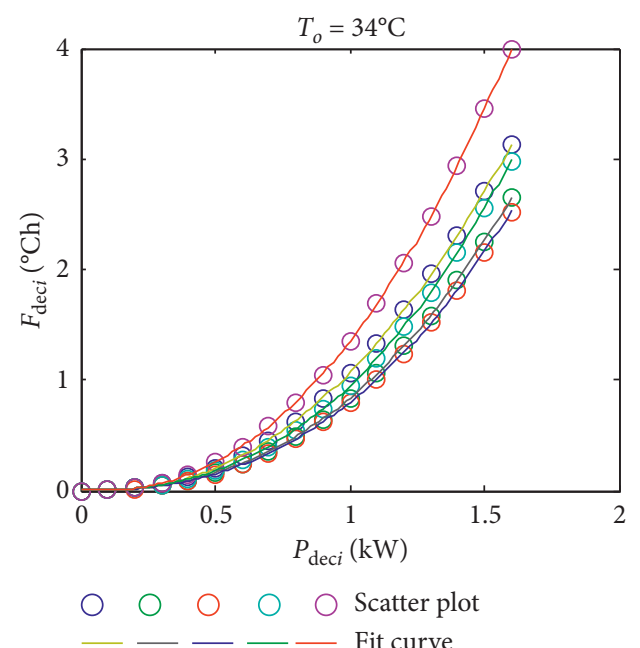

(e)

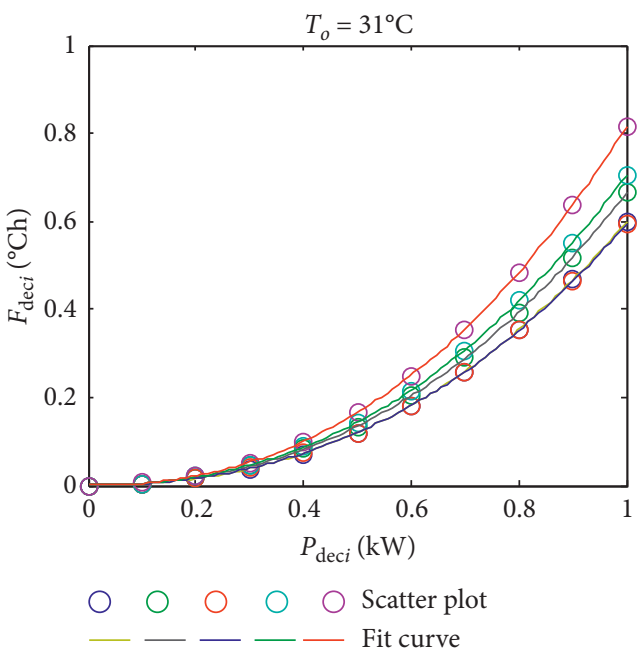

(b)

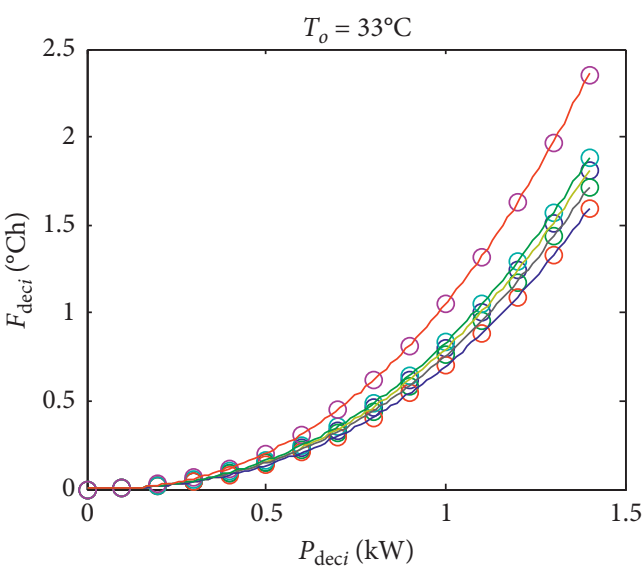

$\bigcirc \bigcirc \bigcirc \bigcirc \bigcirc$ Scatter plot

(d)

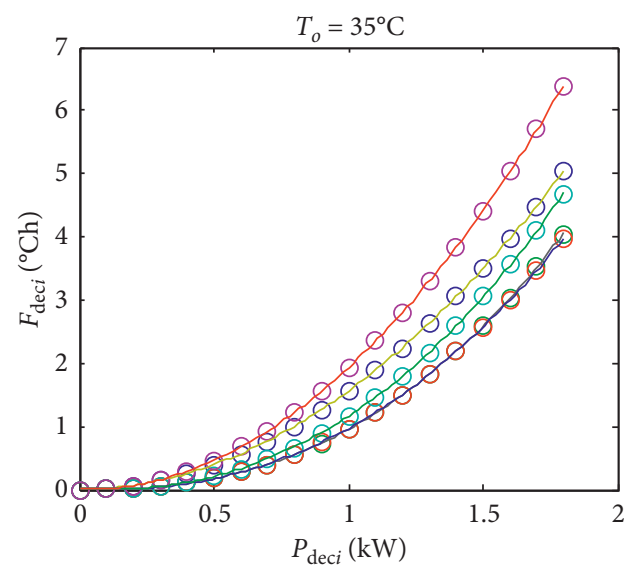

$\because \bigcirc \bigcirc \bigcirc \bigcirc$ Scatter plot

(f)

Figure 7: Scatter plot and fit curve of $P_{\mathrm{dec}}-F_{\mathrm{dec}}$ with different outer temperatures. 

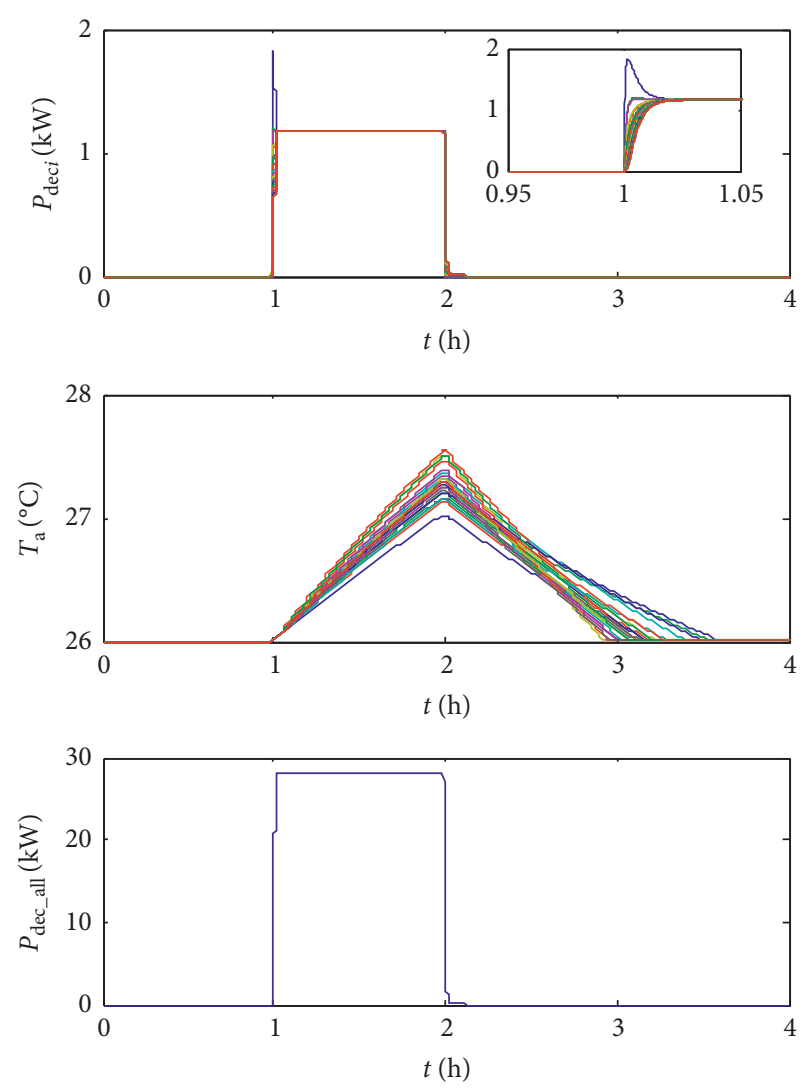

FiguRe 8: Simulation results of method 1.
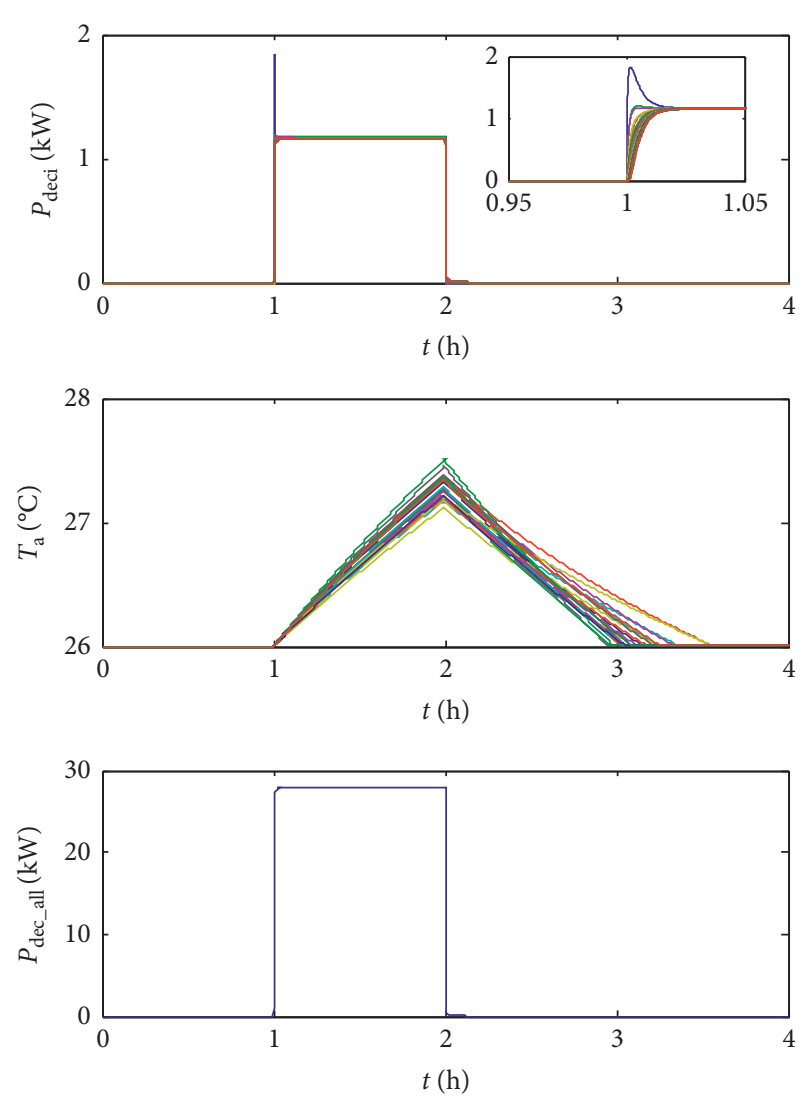

FIGURE 9: Simulation results of method 2.
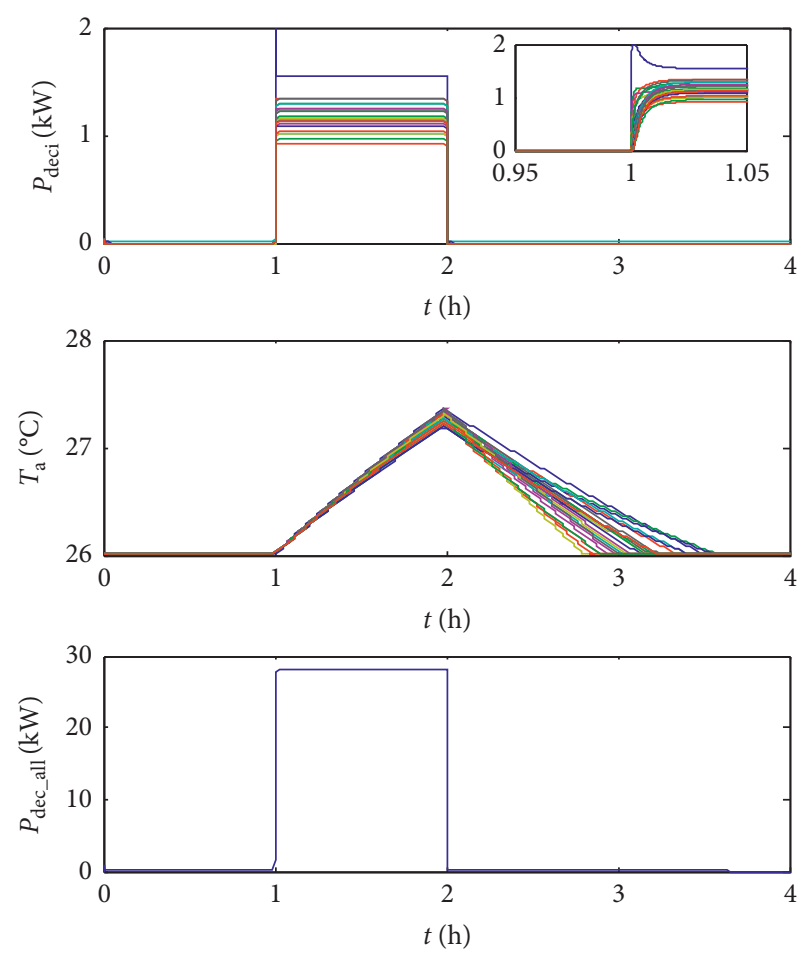

Figure 10: Simulation results of method 3.

TABle 3: Comparison of performance from methods 1-3.

\begin{tabular}{lcc}
\hline & $F_{\text {dec_all }}$ & IAE \\
\hline Method 1 & 29.2038 & 0.1120 \\
Method 2 & 29.5383 & 0.1115 \\
Method 3 & 28.5434 & 0.1047 \\
\hline
\end{tabular}

which is significantly improved compared with method 1 and method 2, thus verifying the effectiveness of the proposed method.

3.1. Results and Discussion. The above testing results verify the effectiveness of the proposed method, including the cost function deriving and the cost function curve fitting, as well as the consensus control strategy. From the analysis of the cost function curve fitting, it can be seen that the third-order polynomial function can fit the cost function very well. By comparing the performance of the proposed method with existing methods, the proposed method exhibits better performance in minimizing the cost.

\section{Conclusion}

Considering the distributed characteristics and the inherent thermodynamic characteristics of air conditioning loads, this paper proposes a multiagent consensus control strategy considering the whole-process thermodynamic characteristics of air-conditioning loads.

The main findings of this paper can be summarized as follows: 
(1) The whole thermodynamic characteristics of air conditioning loads includes two processes: the load reduction process and the recovery process, based on which the power-cost relationship can be established

(2) Third-order polynomial fitting can better reflect the power-cost relationship of air-conditioning load, and the incremental cost consensus control strategy can reflect the cost in the control process and therefore minimize the adjustment cost

Compared with the traditional method, the proposed method has the following advantages:

(1) The cost function can reflect the thermodynamic characteristics of the whole process (reduction process-recovery process) of air-conditioning loads

(2) The multiagent consensus control strategy based on incremental cost can realize the optimal power allocation of air-conditioning loads

Future work may develope a more complex online monitoring and control system for the air conditioners considering the time-varying parameters.

\section{Nomenclature}

Ta: $\quad$ Air temperature in the room

$T_{o}$ : $\quad$ Outdoor temperature

$R: \quad$ Thermal resistance of the room

$C: \quad$ Heat capacity of the room

$P$ : $\quad$ Electrical power of air conditioning load

$\eta$ : $\quad$ Thermal efficiency of air conditioning load

$F_{\text {deci }}: \quad$ Cost of the $i$ th air conditioner

$P_{\text {deci }}$ : Power reduction of the $i$ th air conditioner

$a_{\mathrm{m}}-a_{0}$ : Coefficients of the cost function

$\lambda_{i}: \quad$ State information (incremental cost) of air conditioner $i$

$\delta_{\mathrm{ij}}$ : $\quad$ Element in the sparse iteration matrix

$l_{\mathrm{ij}}$ : $\quad$ Element of the Laplacian matrix

$\varepsilon: \quad$ Convergence coefficient

$P_{\text {dec_req }}$ : Power reduction command of all the air conditioners.

\section{Data Availability}

All data generated or analyzed during this study are included within the article.

\section{Conflicts of Interest}

The authors declare that there are no conflicts of interest.

\section{Acknowledgments}

This study was supported by Science and Technology Project of China Southern Power Grid Corporation (Research on key technologies of flexible load participation in regional power grid dispatching in megalopolis 090000KK52190230).

\section{References}

[1] Y. Bao, P. Chen, and M. Hu, "Control parameter optimization of thermostatically controlled loads using a modified statequeuing model," CSEE Journal of Power and Energy Systems, vol. 6, no. 2, pp. 394-401, 2020.

[2] D. Yang, Z. Lin, and W. Lai, "Air conditioning load control strategy under the background of virtual power plant," Power Demand Side Management, vol. 22, no. 1, pp. 48-85, 2020.

[3] B. Li, Z. Li, and B. Chen, "Air conditioning group control strategy of distribution network in power market," Power System Automation, vol. 43, no. 15, pp. 124-134, 2019.

[4] Z. A. Shah, H. F. Sindi, A. Ul-Haq, and M. A. Ali, "Fuzzy logicbased direct load control scheme for air conditioning load to reduce energy consumption," IEEE Access, vol. 8, pp. 117413-117427, 2020.

[5] B. Li, Z. Li, and B. Chen, "Load control strategy of central air conditioning in Intelligent Park Based on DFT [J]," Power Grid Technology, vol. 44, no. 7, pp. 2549-2559, 2020.

[6] J. J. R. Liu, J. Lam, and K.-W. Kwok, "Further improvements on non-negative edge consensus of networked systems," IEEE Transactions on Cybernetics, vol. 9, no. 1, pp. 1-9, 2021.

[7] J. J. R. Liu, J. Lam, and Z. Shu, "Positivity-preserving consensus of homogeneous multiagent systems," IEEE Transactions on Automatic Control, vol. 65, no. 6, pp. 2724-2729, 2020.

[8] D. Yao, H. Li, R. Lu, and Y. Shi, "Event-triggered guaranteed cost leader-following consensus control of second-order nonlinear multiagent systems," IEEE Transactions on Systems, Man, and Cybernetics: Systems, vol. 10, no. 1, pp. 1-10, 2021.

[9] J. Khazaei and Z. Miao, "Consensus control for energy storage systems," IEEE Transactions on Smart Grid, vol. 9, no. 4, pp. 3009-3017, 2018.

[10] B. N. Alhasnawi, B. H. Jasim, and B. E. Sedhom, "Distributed secondary consensus fault tolerant control method for voltage and frequency restoration and power sharing control in multiagent microgrid," International Journal of Electrical Power \& Energy Systems, vol. 133, no. 2, Article ID 107251, 2021.

[11] B. N. Alhasnawi, B. H. Jasim, B. E. Sedhom, E. Hossain, and J. M. Guerrero, "A new decentralized control strategy of microgrids in the internet of energy paradigm," Energies, vol. 14 , no. 8, p. 2183, 2021.

[12] B. Wang, T. Zhang, X. Hu, Y. Bao, and H. Su, "Consensus control strategy of an inverter air conditioning group for renewable energy integration based on the demand response," IET Renewable Power Generation, vol. 12, no. 14, pp. 16331639, 2018.

[13] K. Meng, D. Wang, D. Wang et al., "Distributed control of thermostatically controlled loads in distribution network with high penetration of solar PV," CSEE Journal of Power and Energy Systems, vol. 3, no. 1, pp. 53-62, 2017.

[14] M. Franceschelli, A. Pilloni, and A. Gasparri, "Multi-agent coordination of thermostatically controlled loads by smart power sockets for electric demand side management," IEEE Transactions on Control Systems Technology, vol. 29, no. 2, pp. 731-743, 2021. 